treatment groups, shown in Figure 1. Disease activity, functional limitations and mood disturbance influenced adherence via multiple and often competing pathways, directly or indirectly through a necessity/concerns evaluation by the patient. Notably, RA activity directly promoted adherence in patients on bDMARDs $(p<0.01)$. In contrast, in patients on csDMARDs, RA activity had an indirect, net negative effect on adherence $(p<0.01)$; the main pathway was through worsening mood disturbance which in turn attenuated adherence $(p<0.01)$. This may imply that the experience of disease activity in patients requiring bDMARDs may be sufficiently impactful to independently promote medication adherence. In contrast, disease activity may be largely experienced as depressed mood in patients on csDMARDs which may in turn overwhelm their capacity to adhere. Additionally, mood disturbance adversely influenced adherence directly in patients on csDMARDs $(p<0.01)$ yet there was no significant direct or net indirect effect of mood disturbance on adherence to biologics. Functional limitations fostered adherence through enhanced awareness of medication necessity $(p<0.05)$.

Conclusion: Distinct domains of the impact of clinical RA influence treatment adherence directly or indirectly through a necessity/concerns framework. Mood disturbance and concerns about medications may represent complementary yet salient intervention targets of comprehensive RA management promoting medication adherence; the former may be more relevant for patients on csDMARDs whereas the latter may be more significant with bDMARDs.

Figure 1: Contributions of clinical RA impact and beliefs about medication on therapeutic adherence.

A. csDMARD treated

Model fit: CFI=.985; $\mathrm{X}^{2}(16)=22.50, \mathrm{p}=.128 ;$ SRMR $=.041 ; \mathrm{RMSEA}=.054$

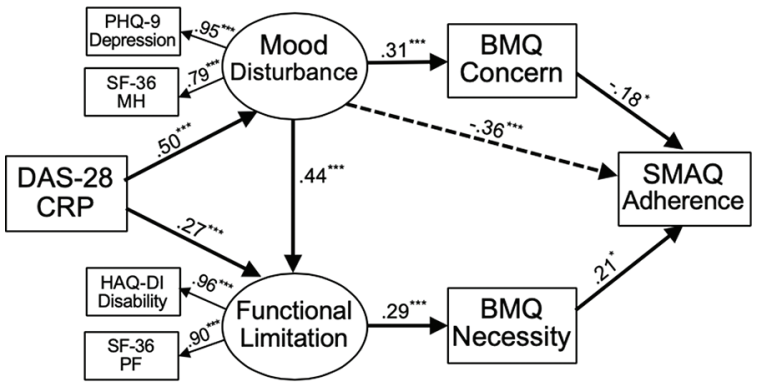

B. bDMARDs

Model fit: CFI $=.999 ; X^{2}(16)=16.34, p=.429 ;$ SRMR $=.037 ;$ RMSEA $=.012$

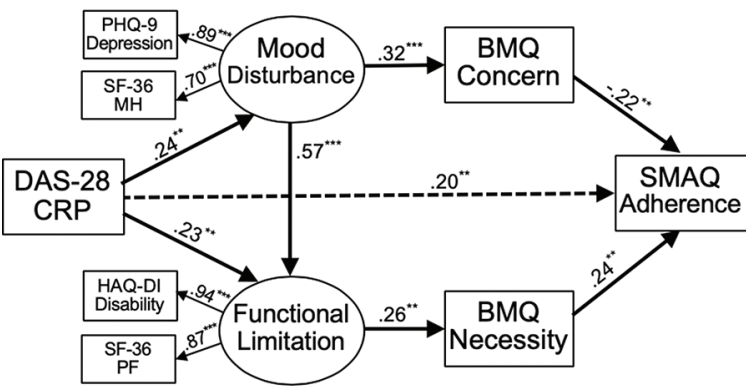

DAS28-CRP: Disease activity score based on 28-joint count and c-reactive protein PHQ-9: 9-item patient health questionnaire, SF-36 MH: Short Form 36 Health Survey mental health scale, HAQ-DI: health assessment questionnaire-disability index, SF-36 PF: Short Form 36 Health Survey physical function scale, BMQ: beliefs about medication questionnaire, SMAQ: Short medication adherence questionnaire. medication questionnaire, SMAQ:
${ }^{*} p<0.05,{ }^{\star \star} p<0.01,{ }^{\star \star \star} p<0.001$

Abstract THU0083 - Figure 1

Disclosure of Interests: George Karpouzas Grant/research support from: Pfizer, Consultant for: Sanofi-Genzyme-Regeneron, Janssen, Roche-Genentech, Pfizer, Speakers bureau: BMS, Sanofi-Genzyme-Regeneron, Janssen, Roche-Genentech, Elizabeth Hernandez: None declared, Vibeke Strand Consultant for: AbbVie, Amgen, Bayer, BMS, Boehringer Ingelheim, Celgene, Celltrion, CORRONA, Crescendo, EMD Serono, Genentech/Roche, GSK, Horizon, Inmedix, Janssen, Kezar, Lilly, Merck, Novartis, Pfizer, Regeneron, Samsung, Sandoz, Sanofi, Servier, UCB., Sarah Ormseth: None declared

DOI: 10.1136/annrheumdis-2019-eular.1222

\section{THU0084 \\ THE LONG-TERM OUTCOME OF INTRA-ARTICULAR GLUCOCORTICOID INJECTED JOINTS ON RADIOGRAPHIC CHANGES IN PATIENTS WITH RHEUMATOID ARTHRITIS: A REAL WORLD DATA FROM A SINGLE CENTER}

Jung Hee Koh ${ }^{1}$, Hanna Lee ${ }^{2}$, Ji-Won Kim³ ${ }^{3}$, Seung Geun Lee ${ }^{2} .{ }^{1}$ Bucheon st.Mary's hospital, The Catholic university of Korea, Division of Rheumatology, Department of Internal Medicine, Bucheon, Korea, Rep. of (South Korea); ${ }^{2}$ Pusan National University Hospital, Division of Rheumatology, Department of Internal medicine, Busan, Korea, Rep. of (South Korea); ${ }^{3}$ Daegu Catholic University Medical Center, Division of Rheumatology, Department of Internal medicine, Daegu, Korea, Rep. of (South Korea)

Background: Intra-articular glucocorticoids injections (IAGI) have been an important part of a treat-to-target strategy in patients with Rheumatoid arthritis (RA). However, it is not known the long-term outcome of glucocorticoids-injected joint.

Objectives: To explore the long-term outcome of IAGI, the impact on the radiographic joint space narrowing and erosion in patients with RA.

Methods: We performed a retrospective cohort study of |AGI for patients with RA. We identified 88 RA patients who received IAGI into wrists or hand joints determined by ultrasound and had pre-injection and follow-up hand radiographs from a rheumatology department in a tertiary hospital. As a comparator, 88 RA patients who had not received IAGI although they had clinical synovitis (defined as both tender and swollen joint) in wrist or hand joint were randomly selected (Non-IAGI group). Hands radiographs scored using the van der Heijde-modified Sharp Score (HSS) method was used to assess radiographic progression ( $\mathrm{HSS} \geq 1$ in joint areas of synovitis). Multivariable Cox regression estimated the hazard ratio $(\mathrm{HR})$ and $95 \%$ confidence interval $(95 \% \mathrm{Cl})$ for radiographic progression of joint with synovitis.

Results: Among 88 RA patients who received IAGI (median age, 60 years, $42(48 \%)$ patients had radiographic progression of triamcinoloneinjected joint, while 38 patients $(43 \%)$ in non-IAGI group (median age, 57 years) presented radiographic progression of joint area suffered clinical synovitis (not significant) in the median 2 years follow-up. At baseline, IAGI group presented longer disease duration, lower acute phase reactant levels and less involvement of proximal interphalangeal joints than nonIAGI group did. In the IAGI group, a median number of 1 (IQR, 1-2) joint was injected. After 3 months from IAGI, $63.5 \%$ of patients reported improvement of injected joint and $16.5 \%$ of patients received a second or more IAGI. The radiographic progression of injected joint (Figure 1) waspronounced at the wrists $(87.8 \%$ of radiographic progressor). The level of C-reactive protein at active arthritis, baseline HSS and power Doppler grade in ultrasound was higher in radiographic progressor than in non-radiographic progressor. A high baseline HSS of hands (HR, $1.021[95 \% \mathrm{Cl}, 1.002-1.040])$, wrist joint involvement (HR, 5.356 [95\% $\mathrm{Cl}, 1.705-16.813])$ and use of NSAIDs (HR, $0.373\left[\begin{array}{ll}95 \% & \mathrm{Cl}, 0.179\end{array}\right.$ $0.776]$ ) were associated with radiographic progression in joint areas which suffered active synovitis.

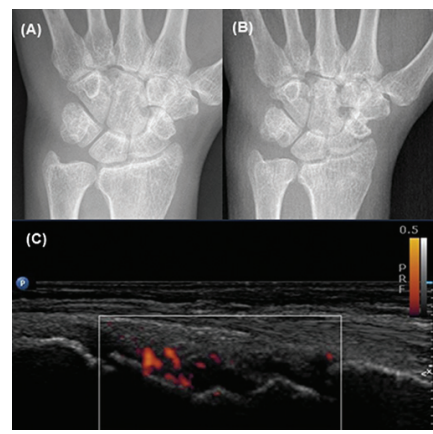

Abstract THU0084 - Figure 1

Conclusion: In patients with RA, IAGI did not have an additional effect on radiographic progression in the next 2 years. High baseline HSS and wrist joint involvement are independent risk factors of radiographic progression in joints suffered synovitis.

\section{REFERENCES:}

[1] Hetland ML, Ostergaard M, Ejbjerg B, Jacobsen S, Stengaard-Pedersen K, Junker P, Lottenburger T, Hansen I, Andersen LS, Tarp U, et al: Shortand long-term efficacy of intra-articular injections with betamethasone 
as part of a treat-to-target strategy in early rheumatoid arthritis: impact of joint area, repeated injections, MRI findings, anti-CCP, IgMRF and CRP. Annals of the rheumatic diseases 2012, 71(6):851-856.

Acknowledgement: This research was supported by grants from the National Research Foundation of Korea (NRF) funded by the Ministry of Science and ICT (NRF-2018R1D1A1B07045491)

Disclosure of Interests: None declared

DOI: 10.1136/annrheumdis-2019-eular.951

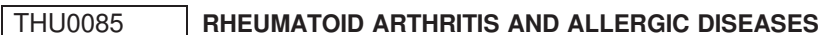

Vanessa Kronzer ${ }^{1}$, Cynthia S. Crowson ${ }^{1}$, Jeffrey Sparks ${ }^{2}$, Robert Vassallo ${ }^{1}$, John Davis ${ }^{1} .{ }^{1}$ Mayo Clinic, Rochester, United States of America; ${ }^{2}$ Brigham and Women's, Boston, United States of America

Background: Historically, RA was considered a $T_{H} 1$ disease while asthma and allergy were considered $\mathrm{T}_{\mathrm{H}} 2$ diseases (1). However, several studies show an association between RA and asthma (2), and more recently, allergic disease (3).

Objectives: We first aimed to determine the association between RA and asthma after controlling for important confounders including allergic disease, urban environment, and passive smoke exposure. Second, we aimed to determine the association between RA and various allergy types.

Methods: This case-control study identified 1,023 cases of RA (175 incident) within a single-center biobank population using a rules-based algorithm that combined self-report with two diagnosis codes. Exposures were self-reported on biobank questionnaires. Logistic regression models calculated the association of exposures with RA, adjusting for potential confounders.

Results: Asthma was associated with RA after adjusting for allergies, urban environment, and passive smoke exposure (OR 1.28, 95\% Cl 1.04 to 1.58). History of allergic disease was also associated with RA (OR $1.30,95 \% \mathrm{Cl} 1.12$ to 1.51 ), especially food allergy (OR 1.38, OR 1.08 to 1.75 , see Figure 1). Results from the incident RA cohort mirrored these findings, though the association with asthma was not significant (OR $1.17,95 \% \mathrm{Cl} 0.66$ to 2.06 )

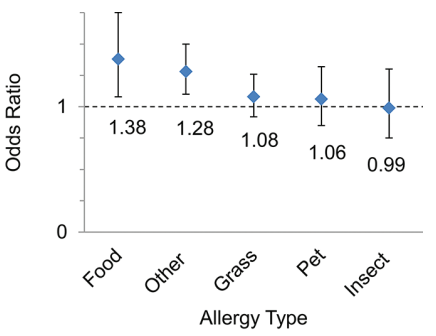

Abstract THU0085 - Figure 1. Adjusted association between allergy types and RA.

Conclusion: Asthma and allergies may be associated with increased risk of RA. It is possible a broader problem with immune dysregulation underlies both RA and allergic diseases, and having one predisposes to the other.

\section{REFERENCES:}

[1] Singh VK, Mehrotra S, Agarwal SS. The paradigm of Th1 and Th2 cytokines: its relevance to autoimmunity and allergy. Immunol Res. 1999;20 (2):147-61.

[2] Sheen YH, Rolfes MC, Wi Cl, Crowson CS, Pendegraft RS, King KS, et al. Association of Asthma with Rheumatoid Arthritis: A Population-Based Case-Control Study. J Allergy Clin Immunol Pract. 2018;6(1):219-26.

[3] Lai NS, Tsai TY, Koo M, Lu MC. Association of rheumatoid arthritis with allergic diseases: A nationwide population-based cohort study. Allergy Asthma Proc. 2015;36(5):99-103.

Acknowledgement: Funding for this project was provided by the Rheumatology Research Foundation Resident Research Preceptorship. We would also like to acknowledge the Mayo Clinic Center for Individualized Medicine

Disclosure of Interests: Vanessa Kronzer: None declared, Cynthia S. Crowson: None declared, Jeffrey Sparks Grant/research support from: Bristol-Myers Squibb, Amgen, Consultant for: Optum, Robert Vassallo: None declared, John Davis Grant/research support from: Pfizer DOI: 10.1136/annrheumdis-2019-eular.4858

\section{THU0086 \\ PREDICTING TNFALPHA INHIBITOR TREATMENT RESPONSE USING SERUM CYTOKINES IN PATIENTS WITH RHEUMATOID ARTHRITIS}

Marissa Lassere ${ }^{1}$, Sue Baker ${ }^{1}$, Jenny Gu ${ }^{2} .{ }^{1}$ UNSW Sydney, St George Hospital SESLHD, Sydney, Australia; ${ }^{2}$ UNSW Sydney, Sydney, Australia

Background: Tumour necrosis factor- $\alpha$ lpha inhibitors (TNF $\alpha$ i) are the main biologics (b-MARDs) used to treat active rheumatoid arthritis (RA) in patients that have failed disease modifying treatment (DMARD). However, $10 \%$ of patients with rheumatoid arthritis, TNF $\alpha$ inhibitors do not work at all. Patients are continued on this treatment for several months risking side-effects in the hope that the treatment will work. Another $40 \%$ of patients respond partially to this treatment and have to also be treated with other drugs such as methotrexate and prednisone in addition to treatment with TNF $\alpha$ i. Biomarkers offer an opportunity to identify before starting or soon after starting treatment with TNFoi which patients will be responders and whether prednisone and other drugs can be reduced and optimise the risk-benefit of treatment.

Objectives: We undertook a series of experiments with the following objectives: determine whether cytokine biomarkers will predict which patients with rheumatoid arthritis are: (a) sustained DMARD early treatment responders (b) sustained TNFoi early treatment responders, (c) TNFoi early treatment failures.

Methods: We used the Millipore's MILLIPLEX MAP Human Th17 Magnetic Bead kit for the simultaneous quantification of the following cytokines: IL-1 $\beta$, IL-2, IL-4, IL-5, IL-6, IL-9, IL-10, IL- 12p70, IL-13, IL-15, IL17A, IL-17E/IL-25, IL-17F, IL-21, IL-22, IL-23, IL-27, IL-28A, IL-31, IL-33, GMCSF, IFN $\gamma, \operatorname{MIP}-3 \alpha, \operatorname{TNF} \alpha$ and TNF $\beta . \quad \gamma, \operatorname{MIP}-3 \alpha, \operatorname{TNF} \alpha$ and TNF $\beta$ We evaluated 14 patients with RA starting on a DMARD and 26 patients with RA starting on a TNF $\alpha$ i after failing DMARDs. These cytokines were assayed monthly 2 or 3 months prior to starting a TNF $\alpha$ i to evaluate month-to-month cytokine variability and every month up to 5 months after initiation of treatment. RA disease activity was measured using the RA Disease Activity Score (DASCRP28) which includes joint counts, CRP and a patient-reported outcome of health status. All samples were blocked with Heteroblock to reduce rheumatoid factor and other heterophilic antibodies. Rheumatoid factor was measured before and after blocking. The same negative and positive controls were included across all plate runs. All assays were done in singlet to accommodate longitudinal samples. Mixing studies were undertaken to evaluate whether cytokine results could be analysed using quantitative statistics.

Results: We had 67 serum samples in the DMARD treated group and 202 serum samples in the TNF $\alpha$ i treated group because of the longitudinal study design. Using mixed effects linear regression to account for longitudinal data in a model that included all 25 cytokines, treatment-time and treatment type (DMARD or TNFoi+/-DMARD), we found that in patients on DMARDs, IL-6, IL-1 $\beta, I L-28 A$, and TNF $\beta$ were associated with treatment response. However, in TNF $\alpha$ i treated patients, TNF-a, GM-CSF and IL- 6 were associated with treatment response. Only $p$ values $<0.005$ are reported given that 25 cytokines were included in the model.

Conclusion: In this study of treatment response comparing DMARDs and TNFoi in a longitudinal cohort of 26 patients with a total of 202 samples measuring TNF $\alpha$ and GM-CSF may predict early TNF $\alpha$ i responders.

Disclosure of Interests: Marissa Lassere Grant/research support from: have received research support with educational grant funding from Sanofi-Aventis, Pfizer, MSD, Abbvie, Sue Baker: None declared, Jenny Gu: None declared

DOI: 10.1136/annrheumdis-2019-eular.6445

\section{THU0087 UTILITY OF INFRARED THERMOGRAPHY FOR THE EVALUATION OF RHEUMATOID ARTHRITIS}

Jesús Loarce-Martos ${ }^{1}$, Javier Bachiller-Corral ${ }^{1}$, Ismael Fernández Cuevas ${ }^{2}$, Manuel Sillero Quintana ${ }^{3}$, Mónica Vázquez Díaz . ${ }^{1} \mathrm{HU}$ Ramón y Cajal, Madrid, Spain; ${ }^{2}$ ThermoHuman, Madrid, Spain; ${ }^{3}$ Universidad Politécnica de Madrid, Madrid, Spain

Background: Rheumatoid Arthritis (RA) is a chronic inflammatory disease which predominantly affects the hands. Currently in clinical practice, we explore the number of swollen and painful joints in order to evaluate the activity of the disease. Infrared thermography (IT) is a non-invasive, lacking ionizing radiation, operator-independent and low-cost technique that allows to measure the temperature of the cutaneous surface through the taking of a photograph.

Objectives: To determine the utility of IT in the evaluation of hands arthritis in patients with RA. 